\title{
LA ENSEÑANZA BILINGÜE EN ANDALUCIA. UN ESTUDIO A PARTIR DE LAS VOCES DE LOS DOCENTES
}

\author{
Gabriel Travé González ${ }^{1}$ \\ Universidad de Huelva. Huelva, España
}

\begin{abstract}
Resumen. El estudio aborda las representaciones del profesorado en torno al bilingüismo y su enseñanza en contextos donde la segunda lengua es extranjera. Se utiliza una metodología integrada combinando datos cuantitativos y cualitativos recogidos a través de un cuestionario administrado a 160 docentes bilingües y entrevistas a un equipo docente. Entre los resultados, la mayoría del profesorado participante en la investigación conceptúa el bilingüismo como el dominio equilibrado de dos lenguas. En cuanto a la finalidad de la enseñanza bilingüe destaca el fomento de la lengua extranjera mayoritariamente, mientras que el tratamiento de las lenguas del alumnado lingüísticamente diverso es rechazado por el $70 \%$ del profesorado. A nivel metodológico se evidencia la predominancia de los enfoques comunicativos, con un mayoritario apoyo del fomento de la comunicación en la lengua extranjera, especialmente entre el profesorado con menor experiencia docente. Los procesos de diseño y desarrollo de la enseñanza bilingüe están fuertemente mediatizados por los libros de texto, que utiliza en su enseñanza ocho de cada diez docentes. Entre las conclusiones, las concepciones del profesorado en torno al bilingüismo siguen referentes que lo conceptúan de forma equilibrada, al tiempo que las finalidades de la enseñanza bilingüe se vinculan con el fomento de la lengua extranjera prioritariamente. Por último, se constata que los procesos de diseño de la enseñanza bilingüe siguen un esquema similar a otras disciplinas del currículo, siendo en la implementación donde se producen las mayores diferencias.
\end{abstract}

Palabras clave: Bilingüismo, Enseñanza bilingüe, Pensamiento del profesorado, Enseñanza Primaria.

\section{BILINGUAL TEACHING IN ANDALUCIA. A STUDY BASED ON TEACHERS' VOICES}

\begin{abstract}
The study analyzes teachers' beliefs on 'bilingualism' and 'bilingual teaching in contexts where the second language is foreign. The research uses integrated methodology combining both quantitative and qualitative data collected in questionnaires administered to 160 teachers and interviews with a team of teachers. Among the results, the majority of teachers conceptualize bilingualism as the equal mastery of two languages. As for the purpose of bilingual teaching teachers largely support the promotion of the foreign language, while treatment of the languages of linguistically diverse student is rejected by $70 \%$ of teachers. With regards to methodology the predominance of communicative approaches is evidenced, with majority support of the promotion of communication in the foreign language, especially among teachers with less teaching experience. Design and implementation of bilingual teaching are strongly mediated by textbooks, which uses in his teaching eight out of ten teachers. Among the conclusions, teachers' beliefs about bilingualism are framed within the balanced visions; while the objectives of bilingual education are linked to the promotion of foreign language.
\end{abstract}

\footnotetext{
${ }^{1}$ Correspondencia: Gabriel Travé González. Campus "El Carmen” Avenida de las Fuerzas Armadas C.P. 21071 Huelva. gabriel.trave@ dedu.uhu.es
} 
Finally, the design processes reflect a scenario in which bilingual teaching receives a similar treatment to other curricular disciplines; being in the implementation where the more differences are to be found.

Keywords: Bilingualism, Bilingual teaching, Teacher cognition, Primary Education.

\title{
EDUCAÇÃO BILÍNGÜE NA ANDALUZIA. UM ESTUDO DA VOZ DOS PROFESSORES
}

\begin{abstract}
Resumo. O estudo aborda as representações dos professores sobre bilingüismo eo ensino em contextos em que a segunda língua é estrangeira. é utilizada uma abordagem integrada da combinação de dados quantitativos e qualitativos recolhidos através de um questionário aplicado a 160 professores e entrevistas bilíngües com um questionário faculdade. Entre os resultados, a maioria dos professores que participam da pesquisa conceitua o bilinguismo como o domínio equilibrada das duas línguas. Quanto à finalidade da educação bilíngüe enfatiza a promoção da língua majoritária estrangeira, enquanto que o tratamento das línguas do estudante diversidade linguística é rejeitada por $70 \%$ dos professores. A nível metodológico a predominância de abordagens comunicativas é evidente, com o apoio da maioria do desenvolvimento da comunicação na língua estrangeira, especialmente entre os professores com menos experiência de ensino. Processos e desenvolvimento do ensino bilingue de design são fortemente mediada por livros didáticos, que usa em seu ensinamento oito em cada dez professores. Entre as conclusões, as concepções de professores sobre o bilingüismo ainda referências que conceituada de forma equilibrada, ao passo que os objectivos da educação bilíngüe estão ligados à promoção de prioridade língua estrangeira. Finalmente, observamos que os processos de projeto de educação bilíngüe seguem um currículo semelhante a outro regime de disciplinas, sendo na aplicação onde as maiores diferenças ocorrem.
\end{abstract}

Palavras-chave: Bilingüismo, educação bilíngüe, o pensamento do professor, Educação Primária.

\section{Introducción}

El fomento del bilingüismo en Europa ha sido uno de las grandes apuestas supranacionales, en un contexto de gran diversidad lingüística en el que se encuentran más de 24 lenguas oficiales, aproximadamente 60 lenguas minoritarias a las que se añaden las lenguas que porta la población inmigrada (Baetens-Beardsmore, 2015).

España, por su parte, representa un claro ejemplo de incorporación de lenguas en el currículo, con realidades diversas en función de la presencia o no de lenguas propias con arraigo comunitario y del grado de apoyo institucional. En Cataluña y País Vasco, por ejemplo, los programas plurilingües llevan implementándose de forma exitosa desde hace años, evidenciándose como fórmulas viables para capacitar al alumnado con competencias lingüísticas en las diferentes lenguas (de Zarobe, 2015).

Andalucía, como nacionalidad histórica monolingüe, ha apostado por el fomento del plurilingüismo fundamentalmente en lenguas de uso internacional. Así, en plena LOGSE se regularizó la anticipación de una lengua extranjera en el segundo ciclo de Educación Infantil y el primer ciclo de Educación Primaria. Asimismo, en el año 2000, 
el Goethe-Institut de Madrid en colaboración con la Junta de Andalucía, puso en marcha una iniciativa encaminada a implantar secciones bilingües alemán-español en escuelas andaluzas. De igual forma, se iniciaron secciones bilingües de lengua francesa a través de acuerdos con el Ministerio de Educación de la República Francesa (Ramos, 2007).

Ya en el año 2005 se aprobó el Plan de Fomento del Plurilingüismo (Consejería de Educación, 2005), orientado a la creación de una estructura institucional para el "Mejorar las competencias lingüísticas de la población andaluza en la lengua materna y, a la vez, dotarla de competencias plurilingües y pluriculturales” (p. 27). Como línea estratégica, se impulsó la creación de una red de centros bilingües que a día de hoy se extiende por toda la geografía andaluza y que en el curso 2014-2015 contaba con 959 centros, integrando -en atención a su volumen- el inglés, francés y alemán, así como el portugués desde el año 2010. La iniciativa contempló desde sus inicios la utilización de la segunda lengua como vehicular en determinadas áreas, materias o módulos profesionales, si bien con diferentes grado de exposición a la lengua según las etapas. Así, en la actualidad, en Educación Infantil se exige la impartición de al menos una hora y media semanal en cada curso, mientras que en Educación Primaria la enseñanza de las áreas de Ciencias de la Naturaleza y Ciencias Sociales ha de realizarse en la segunda lengua (con un margen entre el cincuenta y el cien por cien de la instrucción en dicha lengua). Asimismo, se posibilita el tratamiento bilingüe, en función de los recursos disponibles, de las áreas de Educación para la Ciudadanía y los Derechos Humanos, Cultura y Práctica Digital, Educación Física y Educación Artística (Consejería de Educación, 2015a).

Después de una primera fase de implementación parcial de la enseñanza bilingüe en la que se crearon líneas bilingües y no bilingües, las enseñanzas se han generalizado al conjunto del alumnado de los centros de forma progresiva a raíz de los cambios normativos introducidos en la Orden de 28 de junio de 2011. Como aspecto destacable, los centros cuentan con la asignación de la figura del auxiliar de conversación a tiempo total a partir de catorce unidades bilingües. A nivel metodológico se ha apostado por el enfoque de Aprendizaje Integrado de Contenidos y Lenguas (Consejería de Educación, 2011; 2015b). La generalización de la enseñanza bilingüe al conjunto del alumnado de los centros en los que se desarrollan las enseñanzas supone, por una parte, un logro en cuanto a política de igualdad, si bien por otro representa un reto añadido al ponerse a prueba la capacidad de los programas para responder a la diversidad desde enfoques inclusivos (Pérez Cañado, 2016).

Los programas bilingües desde el enfoque AICLE muestran una gran variabilidad en el contexto europeo, hasta tal punto que ya Coyle (2007) diferenciaba entre 216 tipologías atendiendo a variables como su obligatoriedad, intensidad, edad de comienzo del alumnado, entre otras. Asimismo, Cenoz, Genesee y Gorter (2014) señalan aspectos diferenciadores como el equilibrio entre las lenguas y el contenido, los objetivos propuestos o las características del alumnado. El éxito de los programas y su rápida propagación viene dado por los beneficios lingüísticos que reporta para el 
alumnado, incluso en contextos como el vasco donde coexisten tres lenguas (Lasagabaster, 2008; Merino y Lasagabaster, 2016). Así, Lorenzo, Casal y Moore (2009) detectaron competencias similares al alumnado que había tenido un acceso temprano a la lengua extranjera según el modelo tradicional de lenguas-asignatura (Vez, 2010). También, se ha identificado la incidencia positiva de la enseñanza según el enfoque AICLE en la reducción de la inhibición del alumnado para expresarse en la lengua extranjera (Méndez y Pavón, 2012; Pavón, Prieto y Ávila, 2015), así como el aumento de los niveles de motivación con respecto a aquellos en modelos de enseñanza convencional de lengua extranjera (Lancaster, 2016; Lasagabaster y López-Beloqui, 2015; Lorenzo et al, 2009; Muscarà, 2010; Vollmer, 2010).

Otra de las claves del éxito comunes a los programas radica en el aporte de significatividad al aprendizaje lingüístico, así tendría "más sentido para el alumno estudiar los ecosistemas marinos en inglés como parte del currículo ordinario de Conocimiento del Medio, que tratar los contenidos de los libros de texto de las clases de idiomas, con frecuencia artificiales" (Lorenzo, 2015, p. 70). En este sentido, la enseñanza bilingüe ha incentivado la búsqueda del "mayor número de conexiones interdisciplinares" entre las diferentes áreas (Salaberri, 2009, p. 64), propiciando además la puesta en marcha de iniciativas integradas innovadoras como las descritas en Huertas et al. (2011) o Travé y Travé (2015).

A pesar de los beneficios lingüísticos, la dimensión del aprendizaje de contenidos curriculares representa un ámbito más controvertido. Así, mientras Anghel, Cabrales y Carro (2013; 2016) identifican un efecto negativo en los niveles de adquisición de contenidos del área de Conocimiento del Medio entre el alumnado de los centros bilingües madrileños, Sotoca (2014) encontraba evidencias en el sentido contrario en el mismo contexto. En cualquier caso, el establecimiento de un adecuado equilibrio entre las dimensiones de lengua y contenidos ha sido señalado como uno de los aspectos más complejos y necesarios en el desarrollo de la enseñanza bilingüe según el enfoque AICLE (Dalton-Puffer, Nikula y Smit, 2010). En este sentido, Pavón (2009, p. 71) sostiene que "En el caso de que se prime el objetivo lingüistico sobre el académico se producirá ineludiblemente un empobrecimiento en la adquisición de los contenidos académicos". Al mismo tiempo que los contenidos han de ser cuidados, el desarrollo de la enseñanza bilingüe ha de contemplar la lengua a través de la que estos se abordan (Lyster, 2008). Así, el sentido de los programas reside en aunar el aprendizaje de contenidos y lengua en un mismo proceso, no debiéndose presuponer que la utilización de la segunda lengua como vehículo de enseñanza satisface exclusivamente las necesidades lingüísticas del alumnado, obviando así toda atención a la forma (Lasagabaster, 2014).

Aludiendo al profesorado adscrito a los programas se han detectado mejoras en su motivación y en su desarrollo profesional (Fernández y Halbach, 2011), destacándose su mayoritario apoyo a la enseñanza bilingüe (Travé y Soto, 2014). Sin embargo, la generalización y proyección de la enseñanza bilingüe presenta numerosos desafíos 
derivados de lograr una adecuada capacitación lingüístico-metodológica entre el profesorado (Cuadrado y Sánchez, 2015; Pérez Invernón, 2013). No en vano, éstos desarrollan su actividad en un contexto sujeto a frecuentes oscilaciones normativas y con escasos criterios de selección del profesorado bilingüe, que en todo caso se limitan a cuestiones de índole linguística, relegando a un segundo plano las dimensiones metodológicas (Ortega, 2015). Mencionar, por último, que la propia percepción de sobrecarga laboral del profesorado bilingüe -especialmente el que ha de compatibilizar las tareas de tutoría, enseñanza bilingüe y docencia en la especialidad de lengua extranjera y de los coordinadores de los programas (Mellado y Bolarín, 2015; Mellado, Bolarín y Porto, 2013; Travé, 2013)- puede a la larga representar una limitación en cuanto a la capacidad del profesorado para hacer frente a las exigencias que entraña el desarrollo de la enseñanza bilingüe.

Considerando la creciente difusión y alcance de la enseñanza bilingüe en el sistema educativo andaluz es necesario continuar indagando en sus características a través de las voces de aquellos que la llevan a práctica. Se asume, de este modo, que las concepciones y representaciones del profesorado configuran teorías que inciden en su práctica y viceversa (Borg, 2015), de ahí la necesidad de someterlas a análisis.

La finalidad de este estudio pretende indagar acerca de las concepciones y práctica reflexionada acerca de la enseñanza bilingüe. Más concretamente, los objetivos que guían este trabajo son los siguientes:

1) Analizar las concepciones del profesorado en torno al concepto de bilingüismo y la finalidad de la enseñanza bilingüe.

2) Identificar las representaciones del profesorado en torno a la metodología, diseño y desarrollo de su enseñanza.

\section{Método}

La investigación que se presenta utiliza una metodología integrada en función de instrumentos pertenecientes tanto a la tradición cualitativa como cuantitativa. Se enriquecen, de este modo, las aportaciones que se podrían alcanzar con un solo enfoque, contrarrestando las limitaciones de las estrategias de método único.

Los problemas de investigación que se formulan en esta aportación son, por un lado ¿qué representaciones de bilingüismo posee el colectivo docente? y por otro ¿qué decisiones curriculares orientan su práctica de aula?:

Los instrumentos de investigación, elegidos en función de su capacidad para analizar las declaraciones de los docentes, han sido el cuestionario, la entrevista y el diario del investigador.

En primer lugar, la vertiente cuantitativa del estudio consistió en la administración de un cuestionario al profesorado bilingüe de la provincia andaluza de 
Huelva acerca de sus representaciones y práctica reflexionada. Se ha optado por la utilización del cuestionario, siendo una de las técnicas de investigación más utilizadas en el estudio de las concepciones y práctica reflexionada de los docentes acerca de la enseñanza bilingüe. El instrumento, de naturaleza estructurada, se basó en una escala no comparativa tipo Likert con cuatro opciones de respuesta: muy en desacuerdo, desacuerdo, acuerdo y muy de acuerdo. Durante su diseño se realizó un proceso de pilotaje consistente en la evaluación del instrumento a través de la revisión por expertos y el análisis de los datos obtenidos tras su administración a una pequeña parte de la muestra. Precisamente, la decisión de optar por una escala tipo Likert par fue motivada principalmente por los resultados del pilotaje del instrumento tras el cual, entre otros aspectos, se detectó la necesidad de omitir la opción de respuesta "No sabe/ no contesta", con la finalidad de evitar la indefinición en la respuesta. El cuestionario definitivo integró veintisiete ítems además de once cuestiones para caracterizar a los participantes. El cuestionario se envió a los 181 docentes en activo de Educación Primaria bilingüe de centros públicos de la provincia de Huelva, obteniéndose un retorno de 160 pertenecientes a 32 centros, en los que el 94\% desarrollan proyectos español-inglés. Los datos se analizaron a través de métodos descriptivos utilizando la aplicación ibm® SPSS 19. En primer lugar, se realizó una primera aproximación a los resultados obtenidos a través de un análisis univariado, caracterizando cada ítem a través de porcentajes. Seguidamente, se desarrolló los análisis de comparaciones de medias utilizando preferentemente la prueba de $\mathrm{T}$ muestras independientes y la ANOVA de un factor.

En cuanto a las características socio demográficas de la muestra, el $78 \%$ del profesorado corresponde a mujeres frente al $22 \%$ de hombres respectivamente. Aludiendo a la localización geográfica del profesorado, el $43 \%$ se encuentra en centros de la capital o del área metropolitana seguido por el $28 \%$ de la costa. El $82 \%$ de los docentes desarrolla su actividad docente en la etapa de Educación Primaria, con un 12\% que complementariamente imparte docencia en Educación Infantil. Sólo el 5\% de los docentes desarrolla su actividad exclusiva en el nivel de Educación Infantil, y un 1.3\% en el primer ciclo de Educación Secundaria. Por su parte, la situación predominante se caracteriza por el hecho de que los docentes bilingües desarrollen su docencia en varios cursos simultáneamente, pertenecientes o no a la misma etapa o ciclo. Así, el 34\% de la muestra desarrolla su actividad en el primer curso de Educación Primaria, el 26\% en segundo y tercero, $25 \%$ en cuarto, $26 \%$ en quinto y un $22 \%$ en sexto. En cuanto a la edad del profesorado, siete de cada diez docentes no supera los 45 años. Abordando la experiencia docente, el 56\% del profesorado lleva al menos diez años en la profesión, con un $31 \%$ del profesorado con al menos cinco años de docencia. En cuanto a la experiencia en enseñanza bilingüe, el $61 \%$ del profesorado lleva entre uno y tres años vinculado a los proyectos. Atendiendo a las características de las tareas desempeñadas por el profesorado, el $69 \%$ de los docentes ejerce las labores de tutoría de un grupo de alumnos/as. Asimismo, el 39\% de los docentes desarrolla las tareas de enseñanza de la especialidad del área de Lengua Extranjera. En cuanto al perfil formativo del 
profesorado, el $84 \%$ ha cursado la especialidad de magisterio por Lengua Extranjera (76\% inglés y $8 \%$ francés), mientras que el $16 \%$ ha cursado otras especialidades entre las que están Educación Primaria, Educación Física, Educación Musical, Educación Infantil, Pedagogía Terapéutica, Ciencias Humanas, así como Matemáticas y Ciencias. Por su parte, el 19\% cuenta con los estudios de licenciatura, principalmente Filología Inglesa (7\%) y Psicopedagogía (6\%), al tiempo que la realización de estudios de máster se documenta solo en el 3\% de la muestra, siendo la realización de los estudios de doctorado testimonial con tan sólo un caso. La actualización lingüística ha sido desarrollada por el $13 \%$ de la muestra a partir de las Escuelas Oficiales de Idiomas, con un 2,5\% que ha realizado el título propio de la universidad de Huelva "Experto en docencia inglés". En cuanto a la valoración de las competencias lingüísticas en lengua inglesa, seis de cada diez docentes (64\%) afirman tener competencias equivalentes al nivel B2 del Marco Común Europeo de Referencia para las Lenguas (MCER), denominado Avanzado dentro de la categoría 'usuario independiente', frente al inmediatamente superior $\mathrm{C} 1$ de 'dominio operativo eficaz' (11\%), o al último nivel C2 o maestría (4\%).

En segundo lugar, se llevó a cabo un estudio cualitativo consistente en el análisis de declaraciones de un equipo docente de primer ciclo de Educación Primaria con la finalidad de analizar sus representaciones y práctica reflexionada en torno a la implementación de la enseñanza bilingüe. Se realizaron nueve entrevistas de naturaleza semiestructurada (cinco individuales y cuatro colectivas). La fase de análisis de las entrevistas representó una etapa decisiva consistente en la sistematización y reducción de los datos acumulados, en la que la estructura de categorías se convirtió en una herramienta esencial. El proceso de análisis se realizó a partir del administrador de bases de datos cualitativos Atlas.Ti (6.2), que ha facilitado además las tareas de administración de los datos y haciendo más transparente el proceso. Se ha utilizado una utilidad del citado programa consistente en la redacción de memos analíticos, de especial utilidad para recoger conclusiones de progreso y reflexiones claves en la obtención de las conclusiones parciales del estudio. La redacción de los memos ha permitido la dimensión analítica e interpretativa a lo largo de todo el proceso de trabajo con la unidad hermenéutica, desde los estadios iniciales de codificación hasta la redacción de las conclusiones parciales del estudio. Finalmente, se han redactado un total de treinta y cinco memos con un total aproximado de diez mil palabras. Además, se analizaron veintiún documentos primarios con un total de doscientas noventa y cinco citas a partir de códigos emanados de los contenidos de las categorías. Los resultados provisionales se recogieron en un informe que fue facilitado a las docentes del equipo con la finalidad de que realizaran aportaciones, modificaciones, sugerencias y/o peticiones de rectificación de información, siguiendo así el compromiso ético establecido en el inicio del estudio a partir del formulario de consentimiento en el que se dejó constancia de los términos de la colaboración y sus derechos. 
Seguidamente, se presentan las categorías del estudio. La primera categoría indaga sobre la conceptuación y finalidad del bilingüismo que tiene el profesorado de la muestra, mientras la segunda hace referencia al estudio de la metodología, diseño y desarrollo de la enseñanza bilingüe (Tabla I).

Tabla I: Categorías, contenidos e instrumentos de investigación.

\begin{tabular}{|c|c|c|}
\hline Categorías & Contenidos & Instrumentos investigación \\
\hline $\begin{array}{l}\text { Conceptuación y finalidad del } \\
\text { bilingüismo }\end{array}$ & $\begin{array}{l}\text { Concepto } \\
\text { Finalidad }\end{array}$ & \multirow{2}{*}{$\begin{array}{l}\text { - Entrevistas colectivas } \\
\text { - Entrevistas individuales } \\
\text { - Cuestionario } \\
\text { - Diario del investigador }\end{array}$} \\
\hline $\begin{array}{l}\text { Metodología, diseño y desarrollo de la } \\
\text { enseñanza bilingüe }\end{array}$ & $\begin{array}{c}\text { Metodología } \\
\text { Diseño } \\
\text { Desarrollo }\end{array}$ & \\
\hline
\end{tabular}

Fuente: Propia

La clasificación de los datos y su posterior volcado permitió triangular los instrumentos, personas, declaraciones e informaciones procediendo a su saturación y la creación de un relato con las suficientes evidencias como para garantizar su credibilidad.

\section{Resultados}

Categoría 1. Concepciones acerca del bilingüismo y la finalidad de la enseñanza bilingüe.

Seguidamente presentamos los resultados de la primera categoría de la investigación que aborda las concepciones del profesorado acerca del bilingüismo y su finalidad.

Analizando el concepto de bilingüismo en el profesorado, el 69\% identifica el dominio perfecto de dos lenguas como criterio para conceptuar como bilingüe a un hablante (ítem 1), evidenciando el mayor peso de las conceptuaciones estrictas de bilingüismo. Paradójicamente, un porcentaje similar de profesorado (66.5\%) se considera hablante bilingüe aún con competencias situadas en el nivel intermedio B2 (64\% del profesorado de lengua inglesa y el 37\% de los docentes de lengua francesa), lo que vendría en todo caso a reafirmar la pervivencia de las definiciones tradicionales de bilingüismo. En este sentido, el profesorado del equipo docente en el estudio de declaraciones sostiene que para ser un hablante bilingüe es preciso "dominar perfectamente la competencia lingüística en las dos lenguas” (Maestra 4, 5:4), considerándose la capacidad para simultanear cada lengua como un rasgo característico 
de bilingüismo: "Una persona que es capaz de hablar en varias lenguas sin discriminar en qué lengua está hablando. Puede intercalar y cambiar sin que se dé cuenta de ello" (Maestra 1, 6:2).

Asimismo, se alude a la necesidad de precisar el bilingüismo dentro del contexto escolar, ya que "el bilingüe perfecto se supone que debe tener la misma competencia en todas las lenguas, otra cosa es el contexto escolar" (Maestra 3, 5:2), poniéndose de manifiesto que "el profesorado adapta su definición generalmente estricta al hacer referencia al bilingüismo escolar" (Diario del investigador, 18:3); aspecto se refuerza en la siguiente declaración: “¡Porque aqui no somos bilingües! El hablante bilingüe como ente tiene que tener las mismas competencias en todas las lenguas" (Maestra 2, 5:3). La utilización del calificativo "ente" para referirse al hablante pone de manifiesto la vigencia de la conceptuación del bilingüe como hablante ideal e idealizado. En coherencia, se valora inapropiada la denominación del centro como bilingüe: " $E l$ nombre 'Centro Bilingüe' es ridículo” (Maestra 2, 5:6), siendo considerado por los docentes como un término inexacto para calificar el tipo de enseñanza que se desarrolla en los centros, más cercana al enfoque AICLE en el marco de proyectos lingüísticos de enseñanza bilingüe enriquecida de baja intensidad. Por otra parte, el profesorado rechaza la posibilidad de que su alumnado llegue a ser bilingüe al final de la Educación Obligatoria (64\% del profesorado de la provincia, ítem 2). De nuevo, la utilización de terminologías con profundos sesgos como la de bilingüe podría condicionar la percepción de los logros obtenidos, aspecto sobre el que sería necesario profundizar en futuras investigaciones.

Pasando a analizar las finalidades acerca de la enseñanza bilingüe en el profesorado se identifican dos grandes áreas vinculadas con las dimensiones de lengua y contenidos paralelamente. De este modo, el $79 \%$ del profesorado considera la potenciación de las competencias linguiísticas del alumnado en la lengua extranjera prioritariamente como finalidad fundamental de la enseñanza bilingüe (ítem 4). En este caso, el análisis del ítem a través de las prueba ANOVA de un factor pone de manifiesto una diferencia estadísticamente significativa en torno a la concepción de la finalidad de la enseñanza bilingüe (Tabla II).

Tabla II: Resultado de la comparación de medias ANOVA de un factor.

\begin{tabular}{|l|c|c|c|c|c|}
\hline & $\begin{array}{c}\text { Suma de } \\
\text { cuadrados }\end{array}$ & $g l$ & $\begin{array}{c}\text { Media } \\
\text { cuadrática }\end{array}$ & $\boldsymbol{F}$ & $\begin{array}{c}\text { Nivel de } \\
\text { significación }\end{array}$ \\
\hline Inter-grupos & 8.61 & 4 & 2,153 & 4,035 & \multirow{2}{*}{, $\mathbf{0 0 4}$} \\
\cline { 1 - 4 } Intra-grupos & 82,156 & 154 &, 533 & & \\
\hline Total & 90,767 & 158 & & & \\
\hline
\end{tabular}

Fuente: Propia 
Así, los docentes en las primeras fases de experiencia docente persiguen la potenciación de las competencias linguiísticas del alumnado en la lengua extranjera prioritariamente, como podemos observar seguidamente en los datos recogidos en la Tabla II, además de visualizar en el gráfico 1.

Profundizando un poco más, y a través del contraste post hoc a partir de la aplicación de la prueba de comparaciones múltiples HSD de Tukey, podemos observar que la correlación estadísticamente significativa se produce en el intervalo 0-5 y 21-30 (Tabla III), visualizándose manifiestamente en la figura 1.

Tabla III: Resultado de la prueba de comparaciones múltiples HSD de Tukey.

\begin{tabular}{|c|c|c|c|c|}
\hline $\begin{array}{c}\text { (I) Años experiencia } \\
\text { docente }\end{array}$ & $\begin{array}{c}\text { (J) Años experiencia } \\
\text { docente }\end{array}$ & $\begin{array}{c}\text { Diferencia de } \\
\text { medias }(\mathbf{I}-\boldsymbol{J})\end{array}$ & Error típico & Sig. \\
\hline \multirow{3}{*}{$0-5$} & $6-10$ &, 247 &, 156 &, 508 \\
\cline { 2 - 5 } & $11-20$ &, 270 &, 157 &, 423 \\
\cline { 2 - 5 } & $21-30$ &, $693^{*}$ &, 177 &, $\mathbf{0 0 1}$ \\
\cline { 2 - 5 } & +31 &, 547 &, 343 &, 503 \\
\hline
\end{tabular}

Fuente: Propia

Figura I: Gráfico de la prueba de comparaciones múltiples HSD de Tukey.

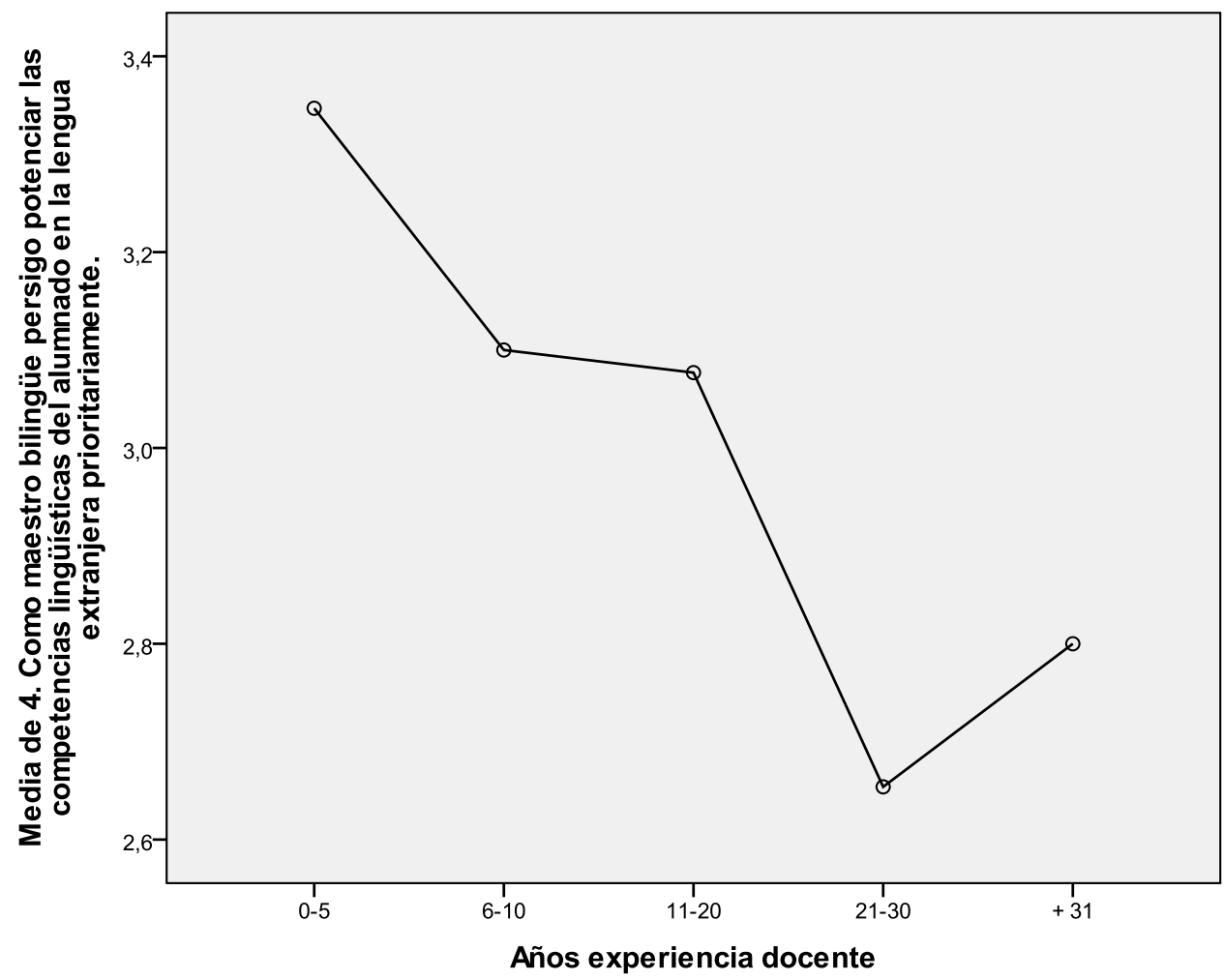

Fuente: Propia 
En esta línea, el conjunto de docentes del equipo considera la potenciación del aprendizaje de la lengua extranjera en el alumnado como finalidad clave de la enseñanza bilingüe: "Un mayor contacto del alumnado con el idioma extranjero. Esto hará que vaya adquiriendo poco a poco, y casi sin darse cuenta, la competencia lingüistica de la forma más natural posible" (Maestra 1, 6:7), y específicamente el desarrollo de la competencia comunicativa: "El objetivo es lograr que el alumnado sea capaz de comunicarse" (Maestra 3, 7:5).

De este modo, se considera la necesidad de promocionar la comunicación frente a la exactitud lingüística, contemplando también los elementos no verbales de todo acto comunicativo: "Me gusta dotar a mi alumnado de las suficientes estrategias para hacerse entender y comprender aunque no lo entiendan todo ni utilicen toda una frase" (Maestra 5, 5:8).

Las concepciones del profesorado acerca de la finalidad de la enseñanza bilingüe pueden ser comprendidas en mayor profundidad a través del análisis de sus representaciones acerca de la posibilidad de que los proyectos incorporen las lenguas del alumnado inmigrante, propuesta que rechazan siete de cada diez docentes (ítem 6). De este modo, el profesorado apoya el fomento de las lenguas prestigiadas y la cobertura de los idiomas con más proyección dentro de una lógica de mercado.

Otra finalidad de la enseñanza bilingüe se orienta al aprendizaje de contenidos a través de una segunda lengua, en clara sintonía con las propuestas derivadas del enfoque AICLE. De este modo, se considera que la enseñanza bilingüe debe potenciar el aprendizaje de contenidos, en un contexto de superación de la enseñanza lingüística desde el enfoque de lenguas-asignatura: "tienen que saber que todos los conocimientos, todas las informaciones pueden venir de diferentes medios y en diferentes lenguas" (Maestra 3, 5:31).

A modo de conclusión, el análisis de la conceptuación de bilingüismo del profesorado refleja que predominan las conceptuaciones equilibradas, evidenciándose la pervivencia del mito del bilingüe como aquel con un dominio perfecto de ambas lenguas. Por su parte, el análisis de las concepciones del profesorado en torno a la potencialidad del programa para lograr sujetos bilingües informa acerca del tipo de concepción que tienen sobre el bilingüismo. De este modo, un amplio sector del profesorado considera que su alumnado no alcanzará competencias suficientes como para denominarlas bilingües al final de la Educación Obligatoria. En este sentido, se hace patente que el término 'bilingüe' introduce connotaciones estrictas, pudiendo reducir la percepción del logro potencial de los proyectos de enseñanza. En cuanto al análisis de las finalidades de la enseñanza bilingüe se pone de relieve la identificación de dos finalidades fundamentales a nivel de lenguas y contenidos. Por una parte, el fomento de la competencia lingüística del alumnado en la lengua extranjera, especialmente la comunicativa; y, por otra, la necesidad de que la enseñanza bilingüe mejore los conocimientos del alumnado. 


\section{Categoría 2. Metodología, diseño y desarrollo de la enseñanza bilingüie.}

El análisis de la categoría 2 se articula sobre el estudio de las concepciones y práctica reflexionada del profesorado en torno a las dimensiones de metodología, diseño y desarrollo de la enseñanza bilingüe.

En líneas generales, la enseñanza bilingüe es concebida por los docentes dentro del enfoque AICLE, si bien se trataría de una apropiación formal consecuencia del uso de una lengua extranjera para la enseñanza de contenidos: "Lo que empecé a atisbar cuando comencé la enseñanza bilingüe es que se basa en entender algunas cosas del área de Conocimiento del Medio en inglés, de forma que el alumnado pueda extraer información de fuentes diversas..." (Maestra 3, 13:1). El análisis de las declaraciones docentes permite poner de relieve que el profesorado manifiesta tener carencias formativas en lo que a enseñanza bilingüe se refiere: "La verdad es que aún no he sido capaz de enterarme muy bien de qué va la enseñanza bilingüe" (Maestra 3, 13:3), limitaciones que especialmente se vinculan con dimensiones de corte metodológico: "La enseñanza bilingüe me produce un poco de cargo de conciencia, de responsabilidad añadida, porque me parece que no sé hacerlo, nunca estoy segura si lo estoy haciendo bien" (Maestra 4, 12:1). A pesar de ello, nueve de cada diez docentes manifiestan haber progresado metodológicamente a raíz de impartir la enseñanza bilingüe (ítem 14).

Los referentes metodológicos del profesorado se enmarcan claramente dentro de los postulados de los enfoques comunicativos, con un $89 \%$ del profesorado convencido de la importancia de fomentar la comunicación del alumnado en la lengua extranjera en el aula durante las sesiones bilingües (ítem 17), como así describe la siguiente docente: "Intento buscar estructuras fáciles y un vocabulario accesible para que puedan entender e interactuar en la lengua 2" (Maestra 3, 13:5). Asimismo, nueve de cada diez docentes respaldan el mayor uso posible de la lengua extranjera por parte del profesorado en las aulas (ítem 21).

Por otra parte, se pone de manifiesto una generalizada superación -al menos a nivel discursivo- de los enfoques expositivos e unidireccionales basados en la actividad del docente, característicos de la metodología directa: "Intento buscar una estructura que el alumnado pueda entender y con la que puedan interactuar y expresarse (...) A lo mejor es un tratamiento del Conocimiento del Medio muy de segunda lengua, que en definitiva es mi terreno y donde me siento cómoda" (Maestra 3, 13:13). De este modo, es perceptible la utilización de elementos metodológicos derivados del área de Lengua Extranjera como así reconoce la siguiente docente: "Utilizo muchas estrategias que pertenecen a mi bagaje como especialista de lengua inglesa (...) Últimamente lo que más resultado me está dando es empezar con ejercicios de actividad física TPR (Total Physical Response)" (Maestra 3, 7:28), hecho que se puede ver fomentado por el propio perfil formativo del profesorado, que en el $84 \%$ de los casos ha cursado la especialidad. 
Haciendo referencia a las características de los procesos de planificación de la enseñanza bilingüe, el 75\% del profesorado afirma que las áreas de Ciencia y Lengua Extranjera se programan conjuntamente (ítem 8). En este sentido, la disciplinariedad se afronta a partir de "una programación lo más integrada posible (...) Tratamos de relacionarlo todo, porque además es antieconómico si lo hacemos todo separado" (Maestra 3, 7:40). Por su parte, se pone de manifiesto que los procesos de diseño de la enseñanza bilingüe se desarrollan de forma colegiada por parte del $63 \%$ del profesorado (ítem 23).

La enseñanza bilingüe se diseña siguiendo las temáticas emanadas del libro de texto, según afirma el 79\% de los docentes (ítem 7): "A partir de los objetivos, contenidos y actividades que están propuestas en el libro de texto analizamos cuáles son más pertinentes, cuáles se pueden adaptar al bilingüe” (Maestra 3, 8:21), utilizando como referente "las orientaciones curriculares oficiales para el primer nivel (currículum) y del libro de texto en cuanto al orden temporal" (Maestra 3, 8:1), evidenciando el papel protagonista de los libros de texto en la selección de las temáticas de estudio. En este sentido, ocho de cada diez docentes lo usa en distintos grados, ya sea como material curricular único o a través de fotocopias de diferentes editoriales. Sin embargo, se caracterizan también prácticas vinculadas al desarrollo de unidades didácticas de elaboración propia y su implementación en diferentes grados de prioridad por parte del $80 \%$ del profesorado. Por otra parte, el proceso de selección de objetivos y contenidos bilingües responde a criterios como la relevancia: "Seleccionamos los objetivos más importantes y llamativos para ser abordados en la lengua extranjera" (Maestra 4, 7:7), la accesibilidad: "Se escogen los contenidos más accesibles y cercanos al alumnado" (Maestra 3, 8:21) y la capacidad para potenciar la comunicación: "El objetivo es lograr que el alumnado sea capaz de comunicarse de forma oral y escrita" (Maestra 3, 7:5).

Analizando el desarrollo de la enseñanza bilingüe, el $79 \%$ del profesorado manifiesta que el desarrollo de la enseñanza bilingüe se aleja de los modelos basados en explicaciones orales y la realización de ejercicios individuales escritos por el alumnado (ítem 16). Por el contrario, las sesiones de clase incorporan una gran diversidad de actividades entre las que se encuentran las destinadas a la explotación de estructuras lingüísticas: "Trabajamos mucho con posters para que el alumnado utilice las estructuras y el vocabulario" (Maestra 4, 7:22); la realización de producciones: "Libros finales de los temas como en el de las aves, en el que hicimos un dibujo y los niños iban describiendo cada animal con frases como Its got a head, long legs..." (Maestra 3, 7:40), además del aprendizaje de canciones "también cuentos, juegos, adivinanzas..." (Maestra 3, 7:21).

El desarrollo aproximado de una sesión de trabajo tipo en el aula consiste en el tratamiento de un poster con la estructura y vocabulario del tema, la participación activa del alumnado y finalmente la realización de una tarea escrita: "Partiendo del poster del tema primero preguntarles si recuerdan el vocabulario para seguidamente invitarles a 
la pizarra para que coloquen las etiquetas con el vocabulario en el poster. Después hacen la ficha en la que tenían que repasar las palabras de la frase” (Maestra 4, 7:60).

También, se afirma realizar actividades con diversos tipos de agrupamiento: "en gran grupo, individuales y por parejas" (Maestra 4, 8:13), reconociéndose que las actividades grupales son menos frecuentes: "no trabajamos demasiado en grupo, hacemos actividades puntuales" (Maestra 2, 14:37). Así, a pesar de valorar la importancia de este tipo de agrupamiento, se encuentran obstáculos para su desarrollo, tanto para el alumnado: "El trabajo en grupo muchas veces plantea conflictos al alumnado, la idea de la colaboración grupal les cuesta" (Maestra 2, 14:38), como para el profesorado: "No sé cómo organizarme bien en las actividades de grupo, ahora los tengo organizados en grupos pero no están trabajando de forma cooperativa" (Maestra $5,14: 38)$.

A modo de resumen de la segunda categoría se pone de relieve una adopción más formal que fundamentada de las bases del enfoque AICLE. En este sentido, el corpus metodológico del profesorado se nutre esencialmente de sus antecedentes formativos en el área de Lengua Extranjera y la propia praxis. En este sentido, a pesar de que se evidencian carencias formativas, el conjunto del profesorado manifiesta haber progresado a nivel metodológico. En cuanto al diseño de la enseñanza bilingüe se realiza una aproximación integrada -al incluir al área de Lengua Extranjera-, y colegiada, ya que se desarrolla en contextos de colaboración entre docentes. La planificación de la enseñanza bilingüe se encuentra fuertemente influenciada por la presencia del libro de texto y el establecimiento de criterios para la selección de objetivos y contenidos basados en la relevancia, la accesibilidad y la capacidad de fomentar la comunicación. Finalmente, los procesos de desarrollo de la enseñanza bilingüe se vinculan con prácticas fundamentadas en la participación activa del alumnado, la comunicación y la realización de diversas tipologías de actividades y agrupamientos que superarían los enfoques directos basados en las exposiciones magistrales y la realización de ejercicios escritos por parte del alumnado.

\section{Discusión y conclusiones}

La investigación desarrollada pone de manifiesto que las concepciones estrictas de bilingüismo son mayoritarias entre el profesorado, al considerar que un hablante sería bilingüe sólo una vez que las lenguas habladas están profundamente enraizadas, reafirmando los resultados obtenidos en un estudio anterior con una muestra parcial en la misma provincia andaluza (Travé, 2013). Se evidencia así que, en esencia, los marcos referenciales del bilingüismo están influenciados por las definiciones tradicionales del campo del aprendizaje de segundas lenguas, que lo han venido a conceptuar como la suma de dos monolingüismos correspondientes bajo el paraguas de un hipotético 'dominio nativo', equilibrado y libre de errores (Valdés, Poza y Brooks, 2015). Paradójicamente, la prevalencia entre el profesorado de concepciones tradicionales y 
escasamente elaboradas, de corte monoglósico, son prevalentes en el marco de proyectos lingüísticos acuñados desde las ideologías heteroglósicas que inspiraron la génesis del plurilingüismo en Europa (de Mejía, 2015). Este sería, por tanto, otro caso más de la divergencia entre los principios que de forma externa se desean difundir en los centros educativos - generalmente a partir de modelos verticales de tipo reformistay las propias concepciones del profesorado que son mucho más resistentes al cambio y sobre las que es necesario intervenir si se pretende mejorar sus prácticas (Borg, 2011; 2015).

Considerando las connotaciones que el término 'bilingüe' tiene entre el profesorado se considera oportuno plantear otras opciones con menores sesgos. Además, la denominación de "centro bilingüe", teniendo en cuenta que la exposición a la lengua extranjera en muchos centros se reduce a una sesión diaria como media, responde más a un tipo de promoción institucional que utilizaría la enseñanza bilingüe como un 'sintagma reclamo' dentro de modelos de excelencia, competitividad y eficacia proyectados hacia el mercado de trabajo y sus exigencias (Díez Gutiérrez, 2015). Desde esta óptica sería positivo apostar por términos como los de 'plurilingües' para los centros -como así ocurre ya en centros en los que se oferta la segunda lengua extranjera a partir del tercer ciclo de primaria- y 'usuarios de la segunda lengua' para los hablantes, así evitando "una excesiva deriva hacia el bilingüismo antes que el plurilingüismo y la interculturalidad" (Galiardo López, 2015). A este respecto, sería más pertinente apostar por el desarrollo de competencias plurilingüies en el alumnado no necesariamente equiparables en las diferentes lenguas, en continua evolución y adaptadas a las necesidades de los usuarios de la lengua a lo largo de sus vidas (Flores y Baetens-Beardsmore, 2015).

$\mathrm{Al}$ indagar en la finalidad que el profesorado otorga a la enseñanza bilingüe se evidencia que los docentes apoyan el fomento de las competencias lingüísticas del alumnado en la lengua extranjera prioritariamente, inglés en la mayoría de los casos, en un marco referencial donde implícitamente se asume una jerarquía de lenguas (Alonso, 2015). Tomando en cuenta la existencia de sesgos a favor de determinadas lenguas se evidencia también una actitud reticente a la incorporación de las lenguas del alumnado lingüísticamente diverso por parte de un profesorado que se presupone sensibilizado con el fomento del plurilingüismo. Este hecho no sólo refuerza las visiones del aprendizaje de lenguas prestigiadas como un aspecto más de las jerarquías culturales occidentales, sino que específicamente confirma una ausencia de reflexión de un sector del profesorado en torno a la necesidad de responder a las realidades progresivamente multilingües en las escuelas y a los derechos lingüísticos del alumnado (SkutnabbKangas, 2015). La constatación de paradojas como esta ha de servir más allá que para reprobar a un profesorado con escasa especialización ya sometido a múltiples presiones en el marco de los centros bilingües (Travé y Soto, 2014), para incentivar su desarrollo profesional, prioritariamente desde vertientes de investigación-acción intercultural. Desde esta lógica, las idealizaciones acríticas de los modelos de éxito social y 
económico que portan consigo las lenguas prestigiadas serían contestadas (Mei Yi Lin, 2008), apostando por perspectivas de enriquecimiento cultural, el fomento del diálogo y la mediación interculturales (Barrios y García, 2005; Byram, 2006; Byram, Gribkova y Starkey, 2002;). Así, es necesario superar los presupuestos asimilacionistas y acríticos desde los que se obvian los derechos del alumnado lingüísticamente diverso y/o la posibilidad de abrir la escuela a otras lenguas (González, Núñez y Trujillo, 2007; Ruiz Bikandi y Tusón, 2006; Galiardo López, 2015), a pesar de que desde la investigación se aportan evidencias de que el reconocimiento y tratamiento de estas lenguas podría favorecer muy positivamente incluso al alumnado lingüísticamente diverso de segunda generación (Medvedeva y Portes, 2016). En este sentido es mucho el camino aún pendiente para hacer de las escuelas espacios de intercambio intercultural (Falcón, Hurtado y Martínez, 2012), considerando que a día de hoy siguen prevaleciendo los enfoques compensatorios basados en la deficiencia donde el alumnado lingüísticamente diverso es atendido fuera del aula (Barrios y Morales, 2012).

Es hora también de reclamar representaciones progresivamente más globales de las finalidades de la enseñanza bilingüe, integrando no sólo el fomento de las diferentes lenguas presentes en el marco escolar -incluidas las del alumnado lingüísticamente diverso- sino el aprendizaje de contenidos relevantes a través de ellas en propuestas que reduzcan la fragmentación curricular y que apuesten por el fomento de enfoques interculturales, tales como las propuestas desde enfoques como el de Aprendizaje basado en Proyectos Globales (Pozuelos, 2007). Así, es necesario considerar la importancia del mensaje, del contenido y del conocimiento frente a los intereses meramente lingüísticos. Hacer de la escuela una academia institucional de idiomas es un riesgo derivado de las visiones hegemónicas que, sin reparos, apuestan por una alfabetización básica, ahora plurilingüe, que dote a la ciudadanía de una capacitación lingüística suficiente para responder a las demandas de un mercado, que si bien ahora demanda el inglés, en pocos años lo dará por consabido pasando a exigir la siguiente lengua representante de la potencia mundial hegemónica (Phillipson, 2009).

Analizando las representaciones del profesorado en torno a la metodología utilizada en la enseñanza bilingüe, se pone de relieve la aceptación del enfoque de Aprendizaje Integrado de Contenidos y Lenguas, en la línea de los resultados obtenidos por Lorenzo et al (2009). De algún modo, estas evidencias vienen a respaldar las consideraciones de Bruton (2011a, 2011b, 2012), quien identifica a los programas AICLE actuales con una nueva expresión de los enfoques comunicativos que ya vivieron su momento álgido en la última década del siglo XX en forma, por ejemplo, del Communicative Language Teaching (CLT) (Richards, 2006).

Sin embargo, se trata más de una apropiación formal -resultante tanto de la utilización de una segunda lengua para la enseñanza de contenidos como de la aplicación de la propia normativa de los programas- que del desarrollo de un cuerpo metodológico propiamente definido y específico para la enseñanza bilingüe. La constatación de carencias formativas en el campo, vuelve a evidenciar la apresurada 
expansión de las enseñanzas en los centros, impulsada sin el acompañamiento ni planificación de medidas para la formación permanente del profesorado a corto-medio plazo, necesidad percibida por el propio profesorado como también evidencia Lancaster (2016). A pesar de la innegable importancia de la dimensión formativa, un análisis de la situación a nivel de formación inicial en Educación Superior vuelve a poner entre las cuerdas la expectativa de contar con graduados adecuadamente formados en enseñanza bilingüe en los próximos años. Así, a día de hoy los grados bilingües siguen siendo minoritarios, habiéndose ofertado de forma experimental una vez la red de centros bilingües era una realidad extendida en la Comunidad Autónoma de Andalucía (Toledo, Rubio y Hermosín, 2012), además de seguir adoleciendo de mecanismos para el seguimiento y monitorización que permitan conocer hasta qué punto se alcanzan las finalidades y objetivos que se persiguen (Jover, Fleta y González, 2016).

Abordando los procesos de diseño es posible constatar la hegemonía de libro de texto como elemento central que determina la configuración curricular de la enseñanza bilingüe. De este modo, teniendo en cuenta que las temáticas se derivan de los manuales en castellano, se limita no sólo su potencialidad para responder a los intereses del alumnado sino el propio margen de acción del profesorado para reflexionar acerca de la selección cultural del conocimiento escolar sobre el que pivotan estas enseñanzas. En este sentido, el diseño de la enseñanza bilingüe sigue la lógica disciplinar en cuanto a la selección y organización del currículo, incorporándose una segunda lengua como vehicular aun manteniendo las estructuras tradicionales inalteradas. Así, si bien la implementación de la enseñanza bilingüe en los centros es difundida como una innovación educativa, el acercamiento al fenómeno desde el análisis curricular revela que en todo caso nos encontramos ante procesos de actualización de la enseñanza (Rodríguez, Pozuelos y García, 2012), en este caso desde una vertiente lingüística.Si el diseño de la enseñanza bilingüe no difiere esencialmente de las prácticas convencionales, su desarrollo a nivel de aula dibuja un escenario más esperanzador caracterizado por la utilización activa de la lengua extranjera, la potenciación de la comunicación y el desarrollo de competencias orales. De este modo, se afirma desarrollar diversas tipologías de actividades y potenciar agrupamientos diversos, si bien el trabajo grupal aún tiene un carácter puntual.

Finalmente, a modo de coda, advertir acerca de la importancia de reclamar una enseñanza plurilingüe innovadora e intercultural en las escuelas. Para ello, es preciso ayudar al profesorado en sus procesos de desarrollo profesional en el campo de la enseñanza bilingüe, comenzando desde las primeras fases de su formación inicial y continuando a través de la formación permanente, además de dotar de recursos adecuados a los centros y mejorar las condiciones laborales del profesorado, sólo así contribuiremos a que esta iniciativa sin precedentes en este contexto se consolide como un referente sostenible en el tiempo y transformador en su esencia. 


\section{Referências}

Alonso, C. S. (2015). Lenguas de comunicación o de negocios.: La aportación contemporánea del bilingüismo crítico a la enseñanza de lenguas. Porta Linguarum: Revista Internacional de Didáctica de las Lenguas Extranjeras, (24), 151-162.

Anghel, B., Cabrales, A. y Carro, J. M. (2013). Evaluación de un programa de educación bilingüe en España: el impacto más allá del aprendizaje del idioma extranjero. En A. Cabrales y A. Ciccone (Coords.) La educación en España: una visión académica, (pp. 53-92). Fedea monografías. Recuperado de http://www.fedea.net/educacion/monografia-2013/web-monografia-educacion2013.pdf

Anghel, B., Cabrales, A. y Carro, J. M. (2016). Evaluating a bilingual education program in Spain: the impact beyond foreign language learning. Economic Inquiry. 54(2), 1202-1223.

Baetens-Beardsmore, H. (2015). Europe: supra-national interventions promoting bilingual education. En P. Mehisto y F. Genesee, Building Bilingual Education Systems (pp. 23-41). Cambridge: Cambridge University Press.

Barrios, Ma.E. y García, J. (2005): "Los programas de enseñanza bilingüe en la educación pública española: el tratamiento de la interculturalidad”. En Luengo, M.R., de la Maya, G. y Gutiérrez, P. (Eds.) Actas del VIII Simposio Internacional de la Sociedad Española de Didáctica de la Lengua y la Literatura "Cultura, Interculturalidad y Didáctica de la Lengua y la Literatura" (pp. 139144). Badajoz: Diputación de Badajoz.

Barrios, Ma.E. y Morales, L. (2012). Apoyo lingüístico inclusivo a alumnado no hispanohablante y aprendizaje de competencias curriculares en Educación Primaria. PORTA LINGUARUM, 17, 203-221.

Borg, S. (2011). The impact of in-service education on language teachers' beliefs. System, 39(3), 370-380.

Borg, S. (2015). Teacher Cognition and Language Education. London: Bloomsbury.

Bruton, A. (2011a). Are the differences between CLIL and non-CLIL groups in Andalusia due to CLIL? Applied Linguistics, 32(2), 236-241.

Bruton, A. (2011b). Is CLIL so beneficial, or just selective? Re-evaluating some of the research. System 39(4), 523-532.

Bruton, A. (2012). What are the real reasons for CLIL? And non-CLIL? Paper presented on 23 March 2012 at the IATEFL Conference. Glasgow: Scotland.

Byram, M., Gribkova, B. y Starkey, H. (2002). Developing the intercultural dimension in language teaching: A practical introduction for teachers. Strasbourg: Council 
of

Europe.

Recuperado

de

https://www.coe.int/t/dg4/linguistic/Source/Guide_dimintercult_EN.pdf

Byram, M. (2006). Developing a concept of Intercultural Citizenship. En G. Alfred, M. Byram y M. Fleming. (Eds.), Education for Intercultural Citizenship: Concepts and Comparisons (pp. 109-129). Clevedon: Multilingual Matters.

Cenoz, J., Genesee, F. y Gorter, D. (2014). Critical analysis of CLIL: Taking stock and looking forward. Applied Linguistics, 35(3), 243-262.

Consejería de Educación (2005). «Acuerdo de 22 de marzo de 2005, del Consejo de Gobierno, por el que se aprueba el Plan de Fomento del Plurilingüismo en Andalucía», en BOJA número 65 de 5 de abril de 2005.

Consejería de Educación (2011). Orden de 28 de junio de 2011, por la que se regula la enseñanza bilingüe en los centros docentes de la Comunidad Autónoma de Andalucía (BOJA núm. 135, martes 12 de julio de 2011).

Consejería de Educación (2015a). Instrucciones de 20 de mayo de 2015 conjuntas de la Dirección General de Innovación Educativa y Formación del Profesorado, y de la Dirección General de Formación Profesional Inicial y Educación Permanente, sobre la organización y funcionamiento de la enseñanza bilingüe para el curso 2015-2016.

Consejería de Educación (2015b). Instrucciones de 1 de junio de 2015, de la Dirección General de Innovación Educativa y Formación del Profesorado, sobre Auxiliares de Conversación para el curso escolar 2015/2016.

Coyle, D. (2007). Content and Language Integrated Learning: Towards a Connected Research Agenda for CLIL Pedagogies, The International Journal of Bilingual Education and Bilingualism, 10(5), 543-562.

Cuadrado, C. y Sánchez, P. (2015). An Analysis and Comparison of the Vocabulary of Teaching Materials: Exploring Bilingual Programmes in Secondary Education. 32nd International Conference of the Spanish Association of Applied Linguistics (AESLA): Language Industries and Social Change. 3-5 April 2014, Seville, SPAIN. Procedia - Social and Behavioral Sciences, 173(13), 61-65.

Dalton-Puffer, C., Nikula, T. y Smit, U. (Eds.) (2010). Language use and language learning in CLIL (AALS Series 7). Amsterdam etc.: Benjamins.

De Mejía, A. (2015). Colombia: Challenges and Constraints. En P. Mehisto y F. Genesee (Eds). Building Bilingual Education Systems (pp. 225-242). Cambridge: Cambridge University Press.

De Zarobe, Y. (2015). Basque country: plurilingual education. Building Bilingual Education Systems. En P. Mehisto y F. Genesee, Building Bilingual Education Systems (pp. 97-108). Cambridge: Cambridge University Press. 
Díez Gutiérrez, E. J. (2015). La construcción educativa del nuevo sujeto neoliberal. Cuadernos de Pedagogía, 454, 76-81.

Falcón, I. G.; Hurtado, I. G. y Martínez, R. G. (2012). Medidas educativas para la atención a la diversidad cultural en la escuela. De la normativa legal a la accesibilidad de información intercultural. Revista Electrónica de Investigación y Docencia (REID), Número Monográfico, Octubre, 2012, 101-123.

Fernández, R. y Halbach, A. (2011). Analysing the situation of teachers in the Madrid Autonomous Community bilingual project. En Y. Ruiz de Zarobe; J. M. Sierra y F. Gallardo (Eds.) Content and Foreign Language Integrated Learning: Contributions to Multilingualism in European Contexts (pp. 241-270). Peter Lang: Bern.

Flores, N. y Baetens-Beardsmore, H. B. (2015). Programs and Structures in Bilingual and Multilingual Education. En W. E. Wright, S. Boun y O. García, The Handbook of Bilingual and Multilingual Education, (pp. 203-222). West Sussex: John Wiley \& Sons.

Galiardo López, A. (2015). Planificación lingüística y enseñanza en Andalucía: la dimensión intercultural dentro del Plan de Fomento del Plurilingüismo. Revista de Llengua I Dret, Journal of Language and Law, 64, 102-126.

González, A., Núñez, Ma. P. y Trujillo, F. (2007). El lugar ideológico de la enseñanza de las lenguas: cuestiones sobre el fenómeno migratorio. Textos de Didáctica de la Lengua y la Literatura, 45,46-59.

Huertas, S., Jiménez, A., León, A., Panadero, F. y De Los Ríos, A. (2011). Una experiencia de AICLE y Currículo Integrado en el IES Alhaken II: "Personnages de Cordoue". E-co: Revista digital de educación y formación del profesorado, 8. Recuperado http://revistaeco.cepcordoba.org/index.php?option=com_content\&view=article\& $\mathrm{id}=135$ :una-experiencia-de-aicle-y-curriculo-integrado-en-el-ies-alhaken-iiqpersonnages-de-cordoueq \& catid=12: monografico \&Itemid=25

Jover, G., Fleta, Ma T. y González, R. (2016). La formación inicial de los maestros de educación primaria en el contexto de la enseñanza bilingüe en lengua extranjera. Bordón. Revista de pedagogía, 68(2), 121-135.

Lancaster, N. (2016). Stakeholder Perspectives on CLIL in a Monolingual Context. English Language Teaching, 9(2), 148-177.

Lasagabaster, D. (2008). Foreign Language Competence in Content and Language Integrated Courses. The Open Applied Linguistics Journal, 1, 31-42.

Lasagabaster, D. (2014). Content versus language teacher: How are CLIL students affected? Utrecht Studies in Language and Communication, 28, 123-141. 
Lasagabaster, D. y López-Beloqui, R. (2015). The Impact of Type of Approach (CLIL Versus EFL) and Methodology (Book-Based Versus Project Work) on Motivation. Porta Linguarum: Revista Internacional de Didáctica de las Lenguas Extranjeras, (23), 41-57.

Lorenzo, F. (2015). Los logros del bilingüismo. Cuadernos de Pedagogía, 458, 69-71.

Lorenzo, F., Casal, S. y Moore, P. (2009). The effects of Content and Language Integrated Learning in European education: Key Findings from the Andalusian Bilingual Sections Evaluation Project. Applied Linguistics, 31(3), 418-442.

Lorenzo, F., Casal, S., Moore, P. y Alfonso, M. (2009). Bilingüismo y educación. Situación de la red de centros bilingües en Andalucía. Centro de Estudios Andaluces. Consejería de la Presidencia.

Lyster, R. (2008). Evolving perspectives on learning French as a second language through inmersion. En D. Ayoun (Ed.). Studies in French Applied Linguistics, (pp. 3-36). Amsterdam: John Benjamins Publishing.

Medvedeva, M. y Portes, A. (2016). Immigrant Bilingualism in Spain: An Asset or a Liability? International Migration Review. Fall, 1-35.

Mei Yi Lin , A. (2008). Cambios de paradigma en la enseñanza de inglés como lengua extranjera: El cambio crítico y más allá." Revista de educación y pedagogía, vol. XX(51), 20-53.

Mellado, M. L. y Bolarín, M. J. (2015). La coordinación en programas bilingües: las voces del profesorado. Aula Abierta, 43(2), 102-109.

Mellado, M. L., Bolarín, M. J. y Porto, M. (2013). Programas bilingües en Educación Primaria: valoraciones de docentes. Porta Linguarum, 20, 253-268.

Méndez, M. y Pavón, V. (2012). Investigating the Coexistence of the Mother Tongue and the Foreign Language through Teacher Collaboration in CLIL Contexts: Perceptions and Practice of the Teachers Involved in the Plurilingual Programme in Andalusia. International Journal of Bilingual Education and Bilingualism, 15(5), 573-592.

Merino, J. A. y Lasagabaster, D. (2015). CLIL as a way to multilingualism. International Journal of Bilingual Education and Bilingualism, 1-14.

Muscarà, M. (2010). Plurilingual education: Teaching and learning in other languages. Procedia Social and Behavioral Sciences, 2, 2902-2905.

Ortega, J. L. (2015). La realidad de la enseñanza bilingüe. Cuadernos de Pedagogía, $458,61-68$.

Pavón, V. (2009). Cambios en la realidad educativa a través de la enseñanza integrada de lenguas y contenidos. En J. Jiménez-Heffernan y M. J. López-Pedraza 
(Coords.), Las lenguas extranjeras como vehículo de comunicación intercultural (pp. 65-84). Madrid: Instituto Superior de Formación del Profesorado.

Pavón, V., Prieto, M. y Ávila, F. J. (2015). Perceptions of Teachers and Students of the Promotion of Interaction Through Task-Based Activities in CLIL. Porta Linguarum: Revista Internacional de Didáctica de las Lenguas Extranjeras, (23), 75-91.

Pérez Cañado, M ${ }^{a}$ L. (2016). From the CLIL craze to the CLIL conundrum: Addressing the current CLIL controversy. Bellaterra Journal of Teaching \& Learning Language \& Literature, Vol. 9(1), 9-31.

Pérez Invernón, M. A. (2013). Evaluación de programas bilingües: análisis de resultados de las secciones experimentales de francés en el marco del Plan de Fomento del Plurilingüismo de Andalucía. Tesis Doctoral. Recuperado de http://helvia.uco.es/xmlui/handle/10396/9359

Phillipson, R. (2009). Linguistic imperialism continued. London: Routledge.

Pozuelos, F. J. (2007). Trabajo por proyectos: descripción, investigación y experiencias. Morón: Ediciones MCEP: Cooperación Educativa.

Ramos, F. (2007). Opiniones de alumnos de un programa bilingüe andaluz sobre su programa y sobre el bilingüismo. Revista Electrónica de Investigación Educativa, 9(2).

Richards, J. (2006). Communicative Language Teaching Today. Cambridge: Cambridge University Press.

Rodríguez, F. P.; Pozuelos, F.J. y García, F. J. (2012). Cuando el cambio llega a la escuela. Estudio de casos sobre los procesos de innovación educativa. Revista Electrónica de Investigación y Docencia (REID), 8, Julio, 2012, 117-141.

Ruiz Bikandi, U. y Tusón, A. (2006). Enseñar y aprender lenguas en el país de acogida. Textos de Didáctica de la Lengua y la Literatura, 42, 8-12.

Salaberri, S. (2009). Un centro y un plan que van de la mano. Cuadernos de Pedagogía, $395,62-65$.

Skutnabb-Kangas, T. (2015). Language rights. En W. E. Wright, S. Boun y O. García (Eds.) The Handbook of Bilingual and Multilingual Education (pp. 185-202). Oxford: John Wiley \& Sons.

Sotoca, E. (2014). La repercusión del bilingüismo en el rendimiento académico en alumnos de colegios públicos de la Comunidad de Madrid. Revista Complutense de Educación, 25(2), 481-500.

Toledo, I., Rubio, F.D. y Hermosín, M. (2012). Creencias, rendimiento académico y actitudes de alumnos universitarios principiantes en un programa plurilingüe. Porta Linguarum, 18: 213-229. [http://hdl.handle.net/10481/15836] 
Travé, G. (2013). Un estudio sobre las representaciones del profesorado de Educación Primaria acerca de la enseñanza bilingüe. Revista de Educación, 361, 379-402.

Travé, G. y Soto, A. (2014). La enseñanza bilingüe según el profesorado de primaria. Características, facilitadores y dificultades. Investigación en la escuela, 84, 7383.

Travé, G. (H) y Travé, G. (2015). Investigamos el mundo del trabajo: una propuesta de investigación escolar en Educación Primaria. Perspectiva Escolar, 381, mayojunio, 12- 18.

Valdés, G., Poza, L. y Brooks, M. (2015). Language Acquisition in Bilingual Education. En W. E. Wright, S. Boun y O. García (Eds.) The Handbook of Bilingual and Multilingual Education (pp. 56-74). Oxford: John Wiley \& Sons.

Vez, J. M. (2010). Didáctica de las lenguas extranjeras: paradigmas de investigación. LINGVARVM ARENA, 1(1), 81-102.

Vollmer, H. (2010). Content and Language Integrated Learning (CLIL): A special case of Language Across the Curriculum (LAC). En C. Bongartz y J. Rymarczyk (Eds.), Languages Across the Curriculum (pp. 27-50). Frankfurt: Peter Lang.

Fecha de recepción: 03/03/2016

Fecha de revisión: 18/05/2016

Fecha de aceptación: 20/06/2016 
Travé, G. 